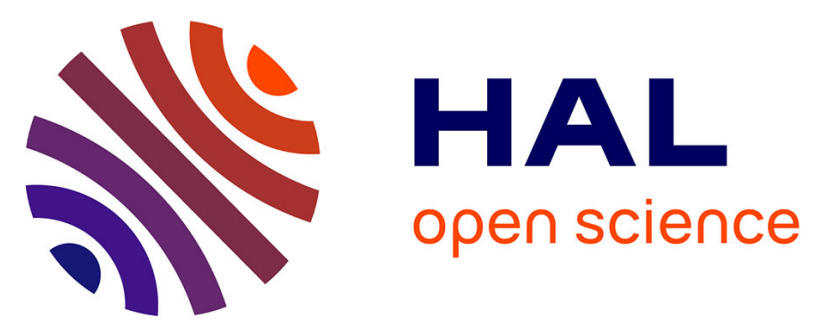

\title{
Spectroscopic ellipsometry: a sensitive tool to monitor domains formation during the bias enhanced nucleation of heteroepitaxial diamond
}

\author{
Julien Delchevalrie, Samuel Saada, R. Bachelet, Guillaume Saint-Girons, \\ Jean-Charles Arnault
}

\section{To cite this version:}

Julien Delchevalrie, Samuel Saada, R. Bachelet, Guillaume Saint-Girons, Jean-Charles Arnault. Spectroscopic ellipsometry: a sensitive tool to monitor domains formation during the bias enhanced nucleation of heteroepitaxial diamond. Diamond and Related Materials, 2021, 112, pp.108246. 10.1016/j.diamond.2021.108246 . hal-03105831

\section{HAL Id: hal-03105831 \\ https://hal.science/hal-03105831}

Submitted on 11 Jan 2021

HAL is a multi-disciplinary open access archive for the deposit and dissemination of scientific research documents, whether they are published or not. The documents may come from teaching and research institutions in France or abroad, or from public or private research centers.
L'archive ouverte pluridisciplinaire HAL, est destinée au dépôt et à la diffusion de documents scientifiques de niveau recherche, publiés ou non, émanant des établissements d'enseignement et de recherche français ou étrangers, des laboratoires publics ou privés. 


\title{
Spectroscopic ellipsometry: a sensitive tool to monitor domains formation during the bias enhanced nucleation of heteroepitaxial diamond
}

J. Delchevalrie ${ }^{1}$, S. Saada ${ }^{1}$, R. Bachelet ${ }^{2}$, G. Saint-Girons ${ }^{2}$, J. C. Arnault ${ }^{1,3 *}$

${ }^{1}$ CEA, LIST, Diamond Sensors Laboratory, 91191, Gif-sur-Yvette, France

${ }^{2}$ INL, CNRS UMR 5270, Université de Lyon, Ecole Centrale de Lyon, F-69134 Ecully, France

${ }^{3}$ NIMBE, UMR CEA-CNRS 3685, Université Paris-Saclay, F-91191 Gif sur Yvette, France

* Corresponding author. Tel: +33 (0)1 690871 02. E-mail: jean-charles.arnault@ cea.fr.

\begin{abstract}
Surface morphology and surface chemistry of epitaxial $\mathrm{Ir} / \mathrm{SrTiO}_{3} / \mathrm{Si}(001)$ pseudo-substrates were systematically characterized by Field Emission Scanning Electron Microscopy (FE-SEM), Atomic Force Microscopy (AFM) and X-ray Photoelectron Spectroscopy (XPS) before and after the Bias Enhanced Nucleation (BEN) of diamond. Based on these characterizations, an ellipsometric model was built taking into account both the roughening of the iridium surface and the formation of domains occurring during the BEN process. Sequential Spectroscopic Ellipsometry (SE) characterizations allowed estimating the roughening of the iridium surface, the thickness of the amorphous carbon layer and the coverage ratio of domains. By annealing the sample in air after BEN, domains were successfully removed without changing the iridium surface morphology. From these analyses, we demonstrate that SE is enough sensitive and discriminative to monitor both the formation of domains and the roughening of iridium surface, distinctly. SE can then be viewed as a powerful in situ tool of critical importance to study, monitor and finally enhance the diamond nucleation and growth on iridium for various advanced applications.
\end{abstract}

\section{Keywords}

Diamond heteroepitaxy, Iridium, Nucleation, Spectroscopic Ellipsometry 



\section{Introduction}

Single crystal diamond presents unique properties, such as a wide bandgap, a high breakdown voltage or a high mobility for both charge carriers. With the possibility to accurately control the doping, this material is a promising candidate for power electronics or radiation detection [1]. However, the size of single crystal diamond synthesized by High Pressure High Temperature (HPHT) or by homoepitaxial growth by Chemical Vapor Deposition (CVD) available for such applications is still limited $\left(<10 \times 10 \mathrm{~mm}^{2}\right)$. Today, diamond heteroepitaxy on iridium appears as a credible alternative to overcome this issue while maintaining a high crystalline quality [2-4].

The few groups involved in diamond heteroepitaxy use iridium surfaces which allows the highest epitaxial rate (> $90 \%$ ). The iridium epilayer is deposited onto scalable substrates [5, 6]. Due to the specific reactivity of iridium under ion bombardment, the nucleation mechanism of diamond differs from the one occurring on silicon and silicon carbide. Indeed, tridimensional isolated crystals are observed on silicon and silicon carbide $[7,8]$ contrary to bidimensional structures obtained on iridium, known as domains [9, 10]. A model including involved mechanisms and kinetics of propagation of domains has been proposed [11]. Sequential approach combining a MPCVD reactor connected to a UHV set-up allowing surface analysis was previously reported to investigate surface modifications induced by Bias Enhanced Nucleation (BEN) on different heterosubstrates [12, 13]. However, in situ characterizations could be much more efficient for having a deeper comprehension of phenomena implied in nucleation. Additionally, it may help the tuning of BEN parameters to improve the quality of diamond / iridium interface. Laser reflectometry has already been investigated to monitor in real time diamond nucleation on silicon. The light scattering induced by the formation of diamond nanocrystals is used to estimate their size and their density (number per $\mathrm{cm}^{2}$ ) [14]. This method is not usable for diamond nucleation on iridium because of the bidimensional structure of domains and of surface modifications induced by the BEN process via a surface roughening (iridium balls, furrows) $[15,16]$. Spectroscopic ellipsometry (SE) could overcome this issue because of its sensitivity to the optical index differences at interfaces. It was already used to deduce in situ the growth rate and the boron concentration in monocrystalline diamond [17] but has never been investigated for diamond nucleation. However, it is necessary to ensure the sensitivity of SE to domains before making in situ characterizations.

In this paper, we report on ex situ characterizations by SE before and after BEN by building an ellipsometric model based on sequential characterizations by X-ray Photoelectron Spectroscopy 
(XPS), Field Emission Scanning Electron Microscopy (FE-SEM) and Atomic Force Microscopy (AFM). By performing annealing in air after BEN to remove domains without affecting the iridium surface morphology, we demonstrate the potential of SE as a sensitive in situ tool to monitor both the iridium surface roughening and the domain formation.

\section{Experimental}

\section{1. $\mathrm{SrTiO}_{3}$ pseudo-substrate on silicon (001)}

The deposition of a thin film of epitaxial $\mathrm{SrTiO}_{3}$ layer on a $500 \mu \mathrm{m}$ thick silicon oriented (001) was achieved by Molecular Beam Epitaxy (MBE) using Knudsen effusion cells. Experimental details can be found elsewhere $[6,18-21]$. A thickness of around $40 \mathrm{~nm}$ has been grown to make further iridium depositions. XRD analysis showed a mosaicity of $0.3^{\circ}$ for the $\mathrm{SrTiO}_{3} / \mathrm{Si}(001)$ heterosubstrates used in this work.

\subsection{Iridium epitaxy on $\mathrm{SrTiO}_{3} / \mathrm{Si}(001)$}

Epitaxial iridium layers were deposited by e-beam evaporation on two $\mathrm{SrTiO}_{3} / \mathrm{Si}(001)$ pseudosubstrates $\left(7 \times 7\right.$ and $\left.10 \times 10 \mathrm{~mm}^{2}\right)[6,22]$. Before the iridium deposition, the substrate is heated at $\sim 400^{\circ} \mathrm{C}$ during $4 \mathrm{~h}$ to remove contaminations. Iridium is then deposited under a pressure below $10^{-6}$ mbar at $680^{\circ} \mathrm{C}$. During the process, the temperature is measured by an infrared pyrometer $(1.45-1.8$ $\mu \mathrm{m})$ with an emissivity of 0.57 . The thickness of the iridium layer was calculated after the deposition by weighting the sample before and after the process with a microbalance (Mettler Toledo MX5), leading to a thickness of $68 \pm 4 \mathrm{~nm}$ for the $7 \times 7 \mathrm{~mm}^{2}$ pseudo-substrate and $84 \pm 4 \mathrm{~nm}$ for the $10 \times 10$ $\mathrm{mm}^{2}$.

\subsection{Bias Enhanced Nucleation of diamond on iridium}

The BEN process was performed in a Microwave Plasma Chemical Vapor Deposition (MPCVD) home-made reactor equipped with a $2 \mathrm{~kW}$ SAIREM microwave generator operating at $2.45 \mathrm{GHz}$. In order to polarize the heterosubstrate during BEN, a DC power supply (Delta Elektronika) delivers a stabilized voltage between 0 and $307 \mathrm{~V}$ associated with a maximal current of $450 \mathrm{~mA}$. A series 
resistance of $15.3 \Omega$ and a Keithley 197A multimeter connected to its terminals allow the measurement of the current in the bias circuit. A counter electrode installed on a translator at $180^{\circ}$ is positively polarized and electrically insulated from reactor walls. In this configuration, the substrate holder is connected to the ground. Nucleation parameters were previously optimized on $7 \times 7 \mathrm{~mm}^{2}$ samples and then adapted to $10 \times 10 \mathrm{~mm}^{2}[6,22]$. BEN parameters i.e. the $\mathrm{CH}_{4}$ vol. $\%$ in $\mathrm{H}_{2}$, the gas pressure $(\mathrm{P})$, the microwave power $\left(\mathrm{P}_{\mathrm{MW}}\right)$, the bias voltage $(\mathrm{U})$, the temperature $(\mathrm{T})$ and the time $(\mathrm{t})$ are presented in the Table 1 for both samples. More details about the nucleation process were reported in our previous works $[4,6,15,23]$.

\begin{tabular}{ccccccc}
\hline $\begin{array}{c}\text { Sample size } \\
\left(\mathbf{m m}^{2}\right)\end{array}$ & $\mathbf{C H}_{\mathbf{4}}$ vol.\% in $\mathbf{H}_{2}$ & $\mathbf{P}(\mathbf{m B a r})$ & $\mathbf{P}_{\mathrm{MW}}(\mathbf{W})$ & $\mathbf{U}(\mathbf{V})$ & $\mathbf{T}\left({ }^{\circ} \mathbf{C}\right)$ & $\mathbf{t}(\mathbf{m i n})$ \\
\hline $7 \times 7$ & 4 & 20 & 600 & -290 & $\sim 700$ & 40 \\
$10 \times 10$ & 4 & 20 & 800 & -290 & $\sim 800$ & 40 \\
\hline
\end{tabular}

Table 1. BEN parameters for $7 \times 7 \mathrm{~mm}^{2}$ and $10 \times 10 \mathrm{~mm}^{2} \mathrm{Ir} / \mathrm{SrTiO}_{3} / \mathrm{Si}(001)$ pseudo-substrates.

\subsection{Characterization techniques}

After each step of the heteroepitaxial diamond synthesis, the sample was observed with a Field Emission (FE)-SEM ZEISS SUPRA 40 equipped with an in-lens detector allowing a lateral resolution of approximatively $3 \mathrm{~nm}$. Using this technique, we are also able to calculate the coverage ratio of domains on the surface after BEN by processing the pictures acquired with ImageJ software. By imaging exactly the same area on the substrate surface, we were also able to compare the iridium morphology before and after the annealing process to remove domains.

AFM analyses were carried out to study the roughening of the iridium surface induced by the BEN. These analyses were done with the contact mode.

XPS analyses of samples before and after BEN were performed using a monochromatized Al Ka anode $(1486.6 \mathrm{eV})$ calibrated versus the $\mathrm{Au} 4 \mathrm{f}_{7 / 2}$ peak located at $84.0 \mathrm{eV}$. The spectrometer was equipped with a hemispherical energy analyser (EA 125, Scienta Omicron, Taunusstein, Germany). The pass energy was $20 \mathrm{eV}$ corresponding to an energy resolution of $0.6 \mathrm{eV}$. The atomic concentrations of iridium, carbon and oxygen were extracted from XPS spectra after corrections by their respective photo-ionization cross-sections. Areas of the corresponding XPS core levels were obtained after a Shirley correction of the background. The fitting of $\mathrm{C} 1 \mathrm{~s}$ core levels was achieved using Voigt 
functions. Assuming a homogeneous carbon layer after BEN, the XPS analysis was also used to extract the thickness of the carbon layer [13].

SE is an optical technique characterization perfectly adapted for studying multi-layer architecture of thin and transparent materials [24]. Ellipsometric spectrometer used in this study is a UVISEL model from Horiba. It is composed of a xenon light as a source, a polarizer, an analyser and two detectors: a photomultiplier for the spectral range from $190 \mathrm{~nm}$ to $520 \mathrm{~nm}$ and an InGaAs photodiode from 520 $\mathrm{nm}$ to $880 \mathrm{~nm}$ at $1 \mathrm{~nm}$ steps with an incidence angle of $70.44^{\circ}$. The connection of these two detectors can induce an artifact at $520 \mathrm{~nm}$. A linear polarized light is reflected on the analysed sample which modifies its polarization state characterized by the ellipsometric angles $\Psi$ and $\Delta$. It is then possible to calculate the parameters $I_{s}$ and $I_{c}$ for each wavelength:

$$
\mathrm{I}_{\mathrm{S}}= \pm \sin (2 \Psi) \sin (\Delta) \quad \mathrm{I}_{\mathrm{c}}= \pm \sin (2 \Psi) \cos (\Delta)
$$

An ellipsometric model created by the user is then compared to experimental data in the $250-825 \mathrm{~nm}$ wavelength range by varying $I_{s}$ and $I_{c}$ deduced from the model in order to extract the thickness, the roughness and the composition of the different layers used in the model. The criterion of a good fit between the model and the experimental data is correlated with the value of $\chi^{2}$ which represents the minimum of a nonlinear error function. This technique potentially allows the characterization of the surface roughening induced by the BEN step (iridium balls, furrows) and domains (formation, coverage ratio).

\section{Results}

In order to make the most complete, simplest and most relevant ellipsometric model, it is required to first characterize the surface morphology and the surface chemistry of samples. For that purpose, FESEM, AFM and XPS analyses were carried out before and after the BEN step. First, the evolution of the surface morphology and the surface chemistry of the pseudo-substrate $\mathrm{Ir} / \mathrm{SrTiO}_{3} / \mathrm{Si}(001)$ is presented, leading to the creation of an ellipsometric model (parts 3.1, 3.2). Second, the experimental data acquired by SE are compared to the simulation coming from the model (part 3.3). Last, we present a way to remove domains without changing the iridium surface morphology for purposes of comparing experimental acquisitions by SE before and after the process on another pseudo-substrate (part 3.4). 


\subsection{Evolution of the surface morphology}

Fig. 1 presents FE-SEM images acquired after the iridium deposition and after the BEN step. In Fig.1a, lines are observable on the iridium surface along the $\langle 110\rangle$ directions. Such structures have been previously reported and are correlated with an epitaxially grown iridium film on a $\mathrm{SrTiO}_{3} / \mathrm{Si}(001)$ pseudo-substrate or $\mathrm{SrTiO}_{3}(001)$ bulk [6]. After the BEN step, domains are formed, identified by areas exhibiting a bright contrast. Iridium balls and furrows are also observed (see enlarged area from Fig. 1b). Thirty images taken in different areas of the surface were processed with ImageJ software to calculate a coverage ratio of $44 \pm 4 \%$ for domains.

(a)

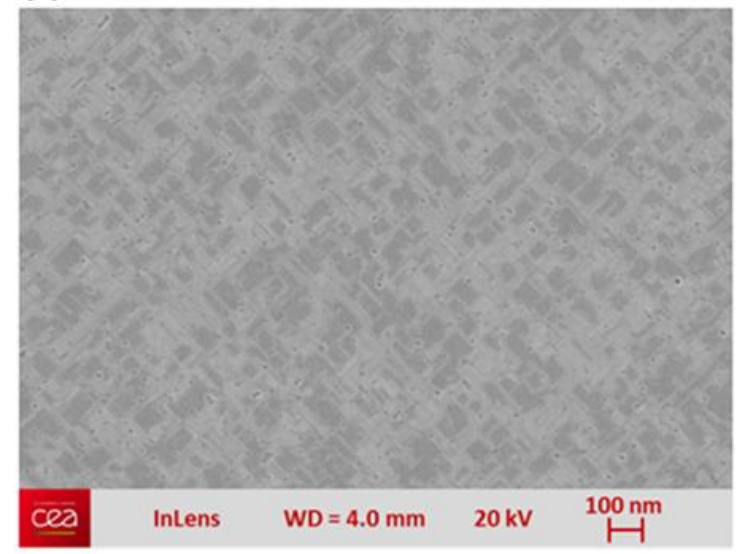

(b)

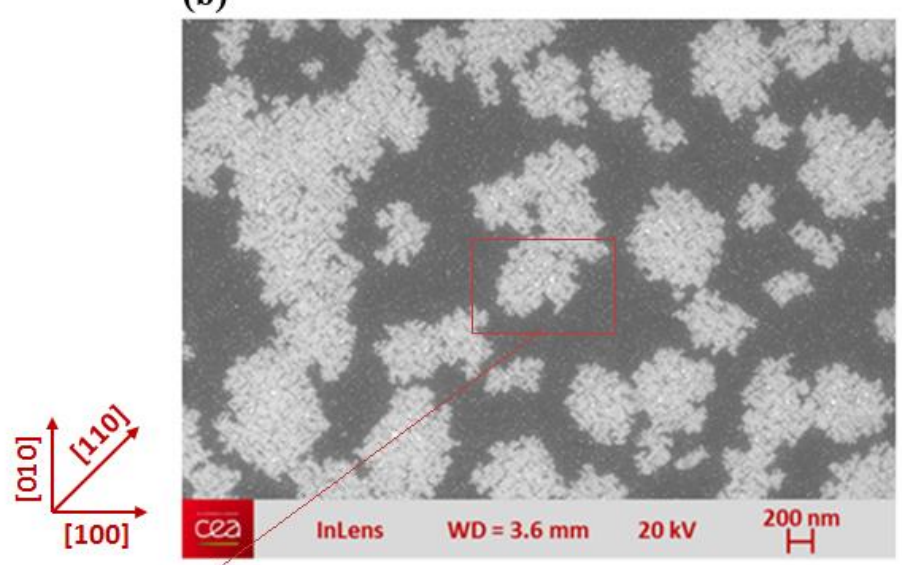

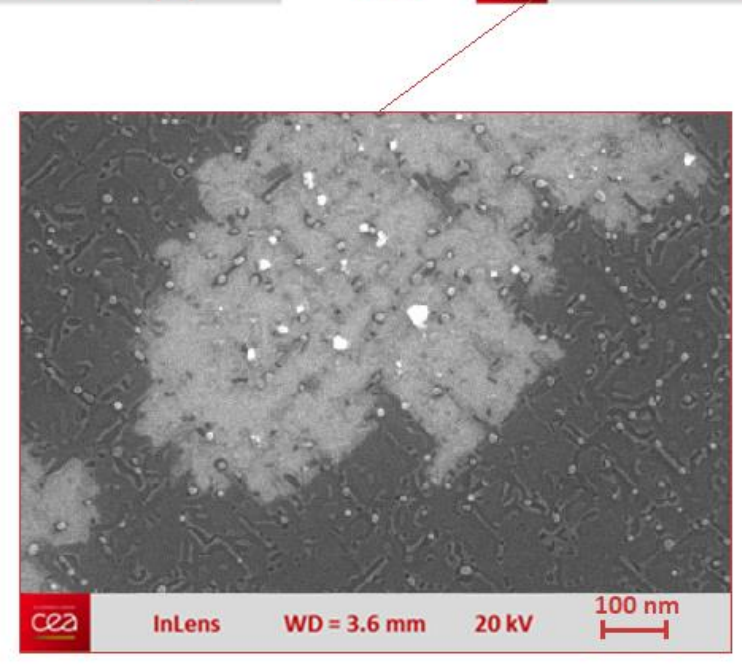

Fig. 1. FE-SEM images of the iridium surface (a) before and (b) after the BEN step. 
AFM images were recorded on large $20 \times 20 \mu \mathrm{m}^{2}$ areas on the center of the sample after the iridium deposition and after the BEN step (Fig. 2). The RMS roughness extracted from these data is $0.7 \mathrm{~nm}$ after the iridium deposition and $2.4 \mathrm{~nm}$ after the BEN step. This increase can be correlated to the formation of iridium balls and furrows observable by FE-SEM (Fig. 1b) but also to diamond nuclei included in domains.
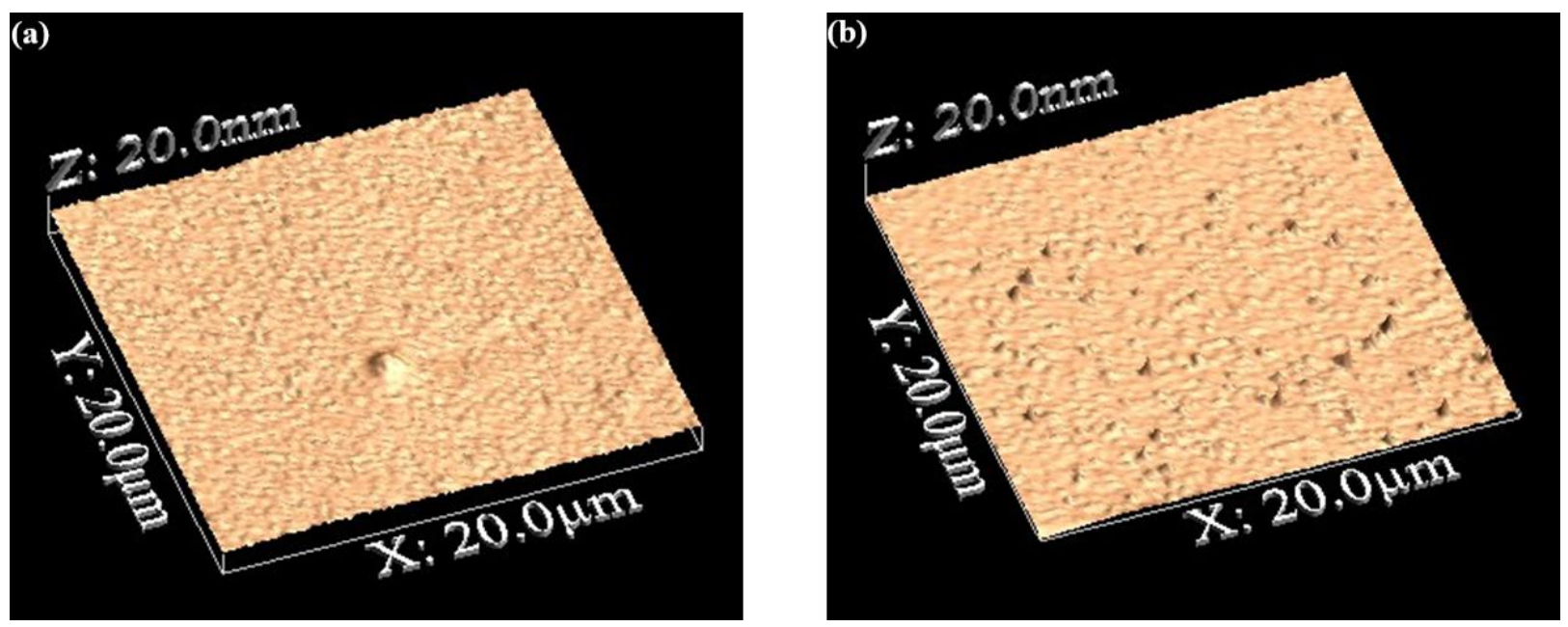

Fig. 2. 3D AFM images of the iridium surface on $20 \times 20 \mu \mathrm{m}^{2}$ (a) before and (b) after the BEN step.

\subsection{Evolution of the surface chemistry}

XPS analysis was conducted before and after the BEN step to characterize the surface modifications occurring during the diamond nucleation by comparing the $\operatorname{Ir}_{4 \mathrm{~d} 5 / 2}$ and $\mathrm{C}_{1 \mathrm{~s}}$ photoemission peaks. Spectra recorded in the 281-301 eV binding energy range are shown on the Fig. 3. The position, the peak full width at half maximum (FWHM) and the atomic concentration of elements present on the surface before and after the BEN step are set out in the Table 2. XPS analysis revealed oxygen and carbon surface contaminations of $27.0 \pm 0.5$ at. $\%$ and $40.0 \pm 0.5$ at. $\%$, respectively, on the initial iridium surface. After the BEN step, we can observe a carbon enrichment of the surface with an atomic concentration of $94.0 \pm 0.5$ at. \% . The oxygen concentration is below the detection limit $(0.5$ at. \%), most likely because of the cleaning induced by a $\mathrm{H}_{2}$ plasma treatment applied before the BEN step $[13,15]$. The distribution of the carbon layer is not obvious to describe. Indeed, after the BEN step, the morphology of the iridium surface strongly evolved with the appearance of iridium furrows and balls and the presence of domains constituted of amorphous carbon and diamond nuclei that partially cover the surface (Fig. 1). The coverage ratio of domains can be quantified by image analysis on FESEM images because it appears with a higher contrast related to a strong electronic emission probably due to the presence of diamond nuclei. On the other hand, the characterization of the domains thickness is more complex because it is of the same order of magnitude compared to iridium roughness. In addition, outside domains, the surface is also covered by a very thin layer of carbon. In a previous publication [15], we proposed a model based on multiple physico-chemical characterizations to give a description of this complex architecture. 
Measurements performed by XPS do not allow to measure the distribution of the carbon layer but can nevertheless be used to characterize the thickness of the equivalent carbon layer assuming an homogeneous layer. Thus, in our study, the equivalent thickness of $4.6 \mathrm{~nm}$ is estimated using the ratio of $\mathrm{C}_{1 \mathrm{~s}}$ and $\mathrm{Ir}_{4 \mathrm{~d} 5 / 2}$ peaks areas which is here of 1.4 .

\begin{tabular}{cccc}
\hline Photoemission peak & Binding Energy $(\mathbf{e V})$ & FHMW $(\mathbf{e V})$ & atomic \% \\
\hline & \multicolumn{2}{c}{ After the iridium deposition } \\
$\mathbf{I r}_{4 \mathrm{~d} 5 / 2}$ & 295.7 & 3.7 & \\
$\mathbf{C}_{\mathbf{1 s}}$ & 283.9 & 1.3 & $33.0 \pm 0.5$ \\
$\mathbf{O}_{\mathbf{1 s}}$ & 535.0 & 3.0 & $40.0 \pm 0.5$ \\
& After the BEN step & $27.0 \pm 0.5$ \\
$\mathbf{I r}_{4 \mathrm{~d} 5 / 2}$ & 295.5 & 3.1 & $6.0 \pm 0.5$ \\
$\mathbf{C}_{\mathbf{1 s}}$ & 283.8 & 1.4 & $94.0 \pm 0.5$ \\
$\mathbf{O}_{\mathbf{1 s}}$ & - & - & $<$ detection limit \\
\hline
\end{tabular}

Table 2. Peak position and FWHM, atomic concentration for elements present on the surface before and after the BEN step.

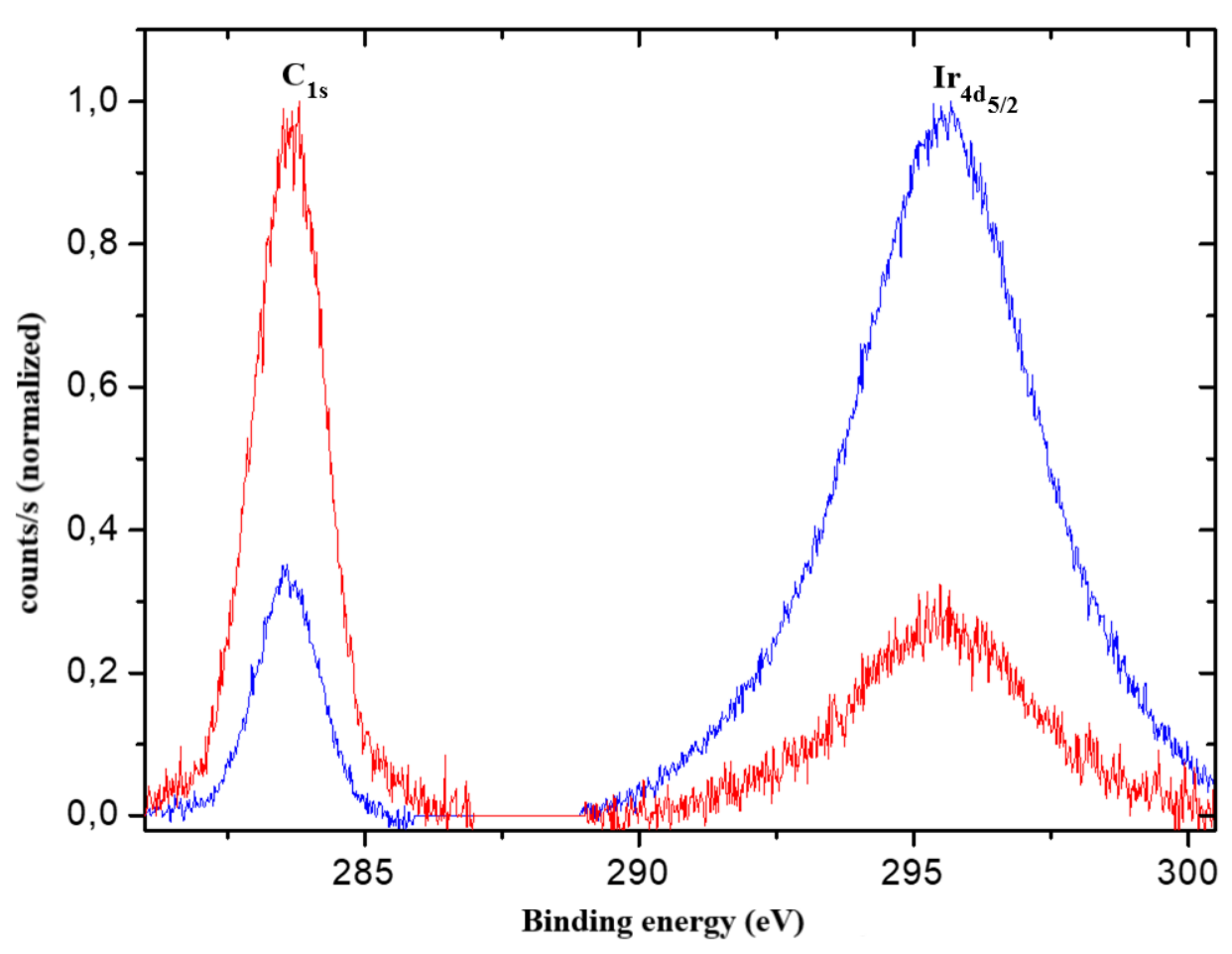


Fig. 3. Comparison of XPS spectra of normalized photoemission peaks of $\operatorname{Ir}_{4 \mathrm{~d} 5 / 2}$ and $C_{1 \mathrm{~s}}$ before (in blue) and after (in red) the BEN step. The spectrum in blue is normalized to the line $\mathrm{Ir}_{4 \mathrm{~d} 5 / 2}$ at $295.7 \mathrm{eV}$ and the spectrum in red is normalized to the line $\mathrm{C}_{1 \mathrm{~s}}$ at $283.8 \mathrm{eV}$. Shirley correction of the background was done for both peaks separately.

These analyses conducted by FE-SEM, AFM and XPS lead to a better description of the evolution of the iridium surface occurring during BEN. We were able to calculate a coverage ratio of domains of $44 \pm 4 \%$ with FE-SEM, bring to light a roughening of the iridium surface by AFM (0.7 nm and 2.4 $\mathrm{nm}$ before and after BEN respectively) and an enrichment of carbon by XPS (40.0 $\pm 0.5 \%$ and $94.0 \pm$ $0.5 \%$ of carbon before and after BEN respectively). XPS analysis also allowed us to estimate a mean thickness of the carbon layer of $4.6 \mathrm{~nm}$. The challenge is now to build an ellipsometric model that is coherent with these physical and chemical characterizations.

\subsection{Spectroscopic Ellipsometry characterizations}

The characterization of iridium surface after the BEN step by SE is relevant because this technique is sensitive to surface morphology, interfaces and optical properties of materials. The main objective is to evaluate this technique to probe domains formation. However, to separate the contribution from iridium surface roughness and domains formation, a multilayers model must be created and must be coherent with the other characterizations of the layers (FE-SEM, XPS and AFM in our study). The $\mathrm{SrTiO}_{3}$ layer deposited on silicon is covered by an iridium layer with a thickness higher than $50 \mathrm{~nm}$. We have previously demonstrated that an iridium layer with a thickness higher than $50 \mathrm{~nm}$ is totally reflective in our case [22]. So, the substrate composed of three layers $\left(\mathrm{Si}_{/} / \mathrm{SrTiO}_{3} / \mathrm{Ir}\right)$ is modelized by an infinite layer of iridium. The optical function of iridium is described by the Tauc Lorentz model function (Fig. 4a). Then, the formation of domains is modelized by a layer of amorphous carbon of homogeneous thickness with an associated porosity to take into account the surface coverage which is not total. The optical function of amorphous carbon is described by an amorphous model function (Fig. $4 b)$. The refraction index $n$ and the extinction coefficient $k$ are constant for both models. The presence of diamond nuclei inside the amorphous carbon layer is neglected. We apply the approximation of effective media that describes, for each layer, the macroscopic optical properties and does not take into account the inhomogeneity of materials and the materials distribution on the surface [25]. 
(a)

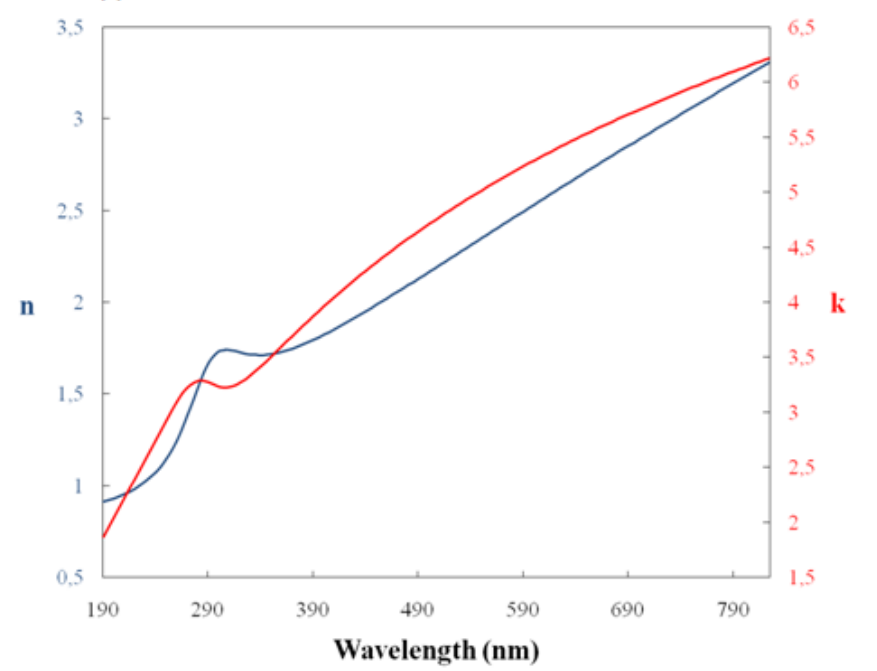

(b)

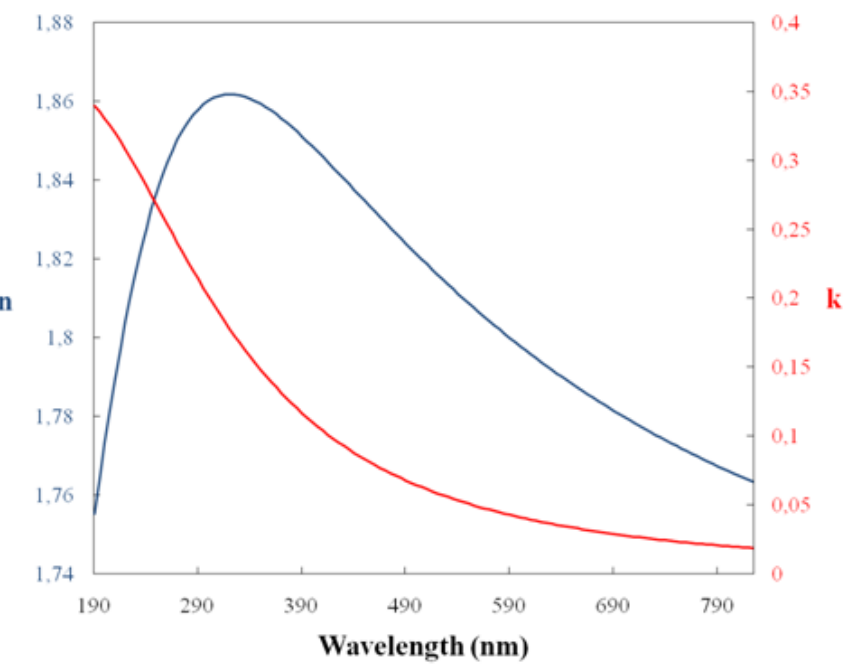

Fig. 4. Optical index $n$ and wave number $k$ for (a) iridium and (b) amorphous carbon.

We compared the experimental data $\left(\mathrm{I}_{\mathrm{s}}, \mathrm{I}_{\mathrm{c}}\right)$ with a first model considering amorphous carbon and void without any contribution from iridium roughness (Fig. 5a). The levels of freedom are limited to the thickness of the amorphous layer and the percentage of void. The model results after optimization are that amorphous carbon composes $100 \%$ of the layer which is $2.4 \pm 0.1 \mathrm{~nm}$ thick. This coverage ratio value is not coherent with the coverage ratio calculated by FE-SEM (44\%). However, the thickness of $2.4 \mathrm{~nm}$ is not too far from the estimated thickness given by XPS (4.6 nm). We observe that this model does not fit accurately the experimental data in the range $250-500 \mathrm{~nm}$ and beyond $700 \mathrm{~nm}$. The slight increase in the $\mathrm{I}_{\mathrm{s}}$ intensity at $520 \mathrm{~nm}$ has no physical meaning because it is correlated with the transition from the first detector to the second one in the ellipsometric spectrometer. The extracted $\chi^{2}$ value for this model is 0.85 . So, this first model is not coherent with the measured coverage ratio of the layer and it does not take into account the iridium surface roughness.

Before combining iridium surface roughness and amorphous carbon layer, we first explore another model, just considering the iridium roughness without domains (Fig. 5b). The roughness is integrated in the model using a layer of iridium with porosity coupled with the approximation of effective media. The levels of freedom are limited to the thickness of the top iridium layer and the percentage of void. After optimization, the best fit is obtained with an iridium layer composed of $50 \pm 2 \%$ of iridium with a thickness of $2.5 \pm 0.1 \mathrm{~nm}$. The iridium thickness is in the same order of magnitude compared to the RMS roughness which is $2.4 \mathrm{~nm}$ over $20 \mathrm{x} 20 \mu \mathrm{m}^{2}$ according to AFM. This second model does not fit precisely the experimental data in the $250-500 \mathrm{~nm}$ range for $I_{\mathrm{s}}$ and $250-350 \mathrm{~nm}$ range for $I_{\mathrm{c}}$. Also, the fit is not perfect beyond $700 \mathrm{~nm}$ for $\mathrm{I}_{\mathrm{s}}$ and $\mathrm{I}_{\mathrm{c}}$. However, the $\chi^{2}$ value calculated for this model is better 
than the previous one: 0.48 . The fit quality is improved but the coherence with the experimental data is insufficient because the carbon layer probed by XPS is not taken into account.

We decided to build a third model combining surface roughening and domain formation through an amorphous carbon layer. It is composed of three layers: the bulk iridium, a layer composed of iridium and amorphous carbon to represent the iridium roughening and the amorphous carbon layer deposition; then a layer made of amorphous carbon and void to represent the coverage ratio of domains (Fig. 5c). 
(a)

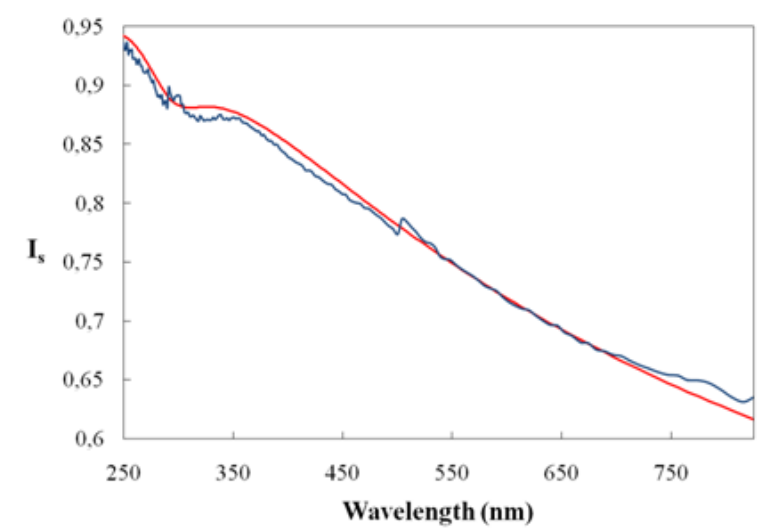

(b)

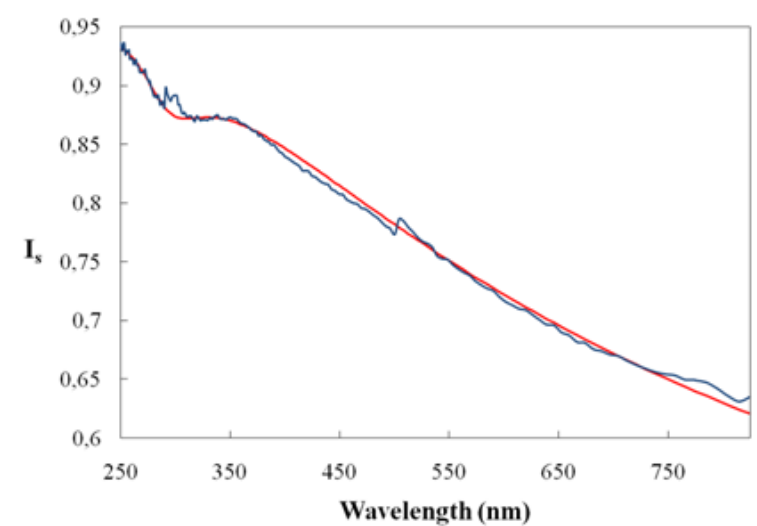

(c)

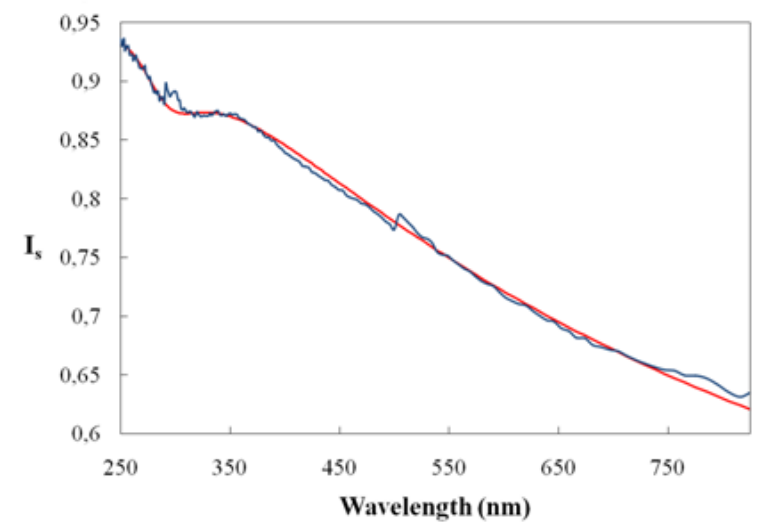

Ir

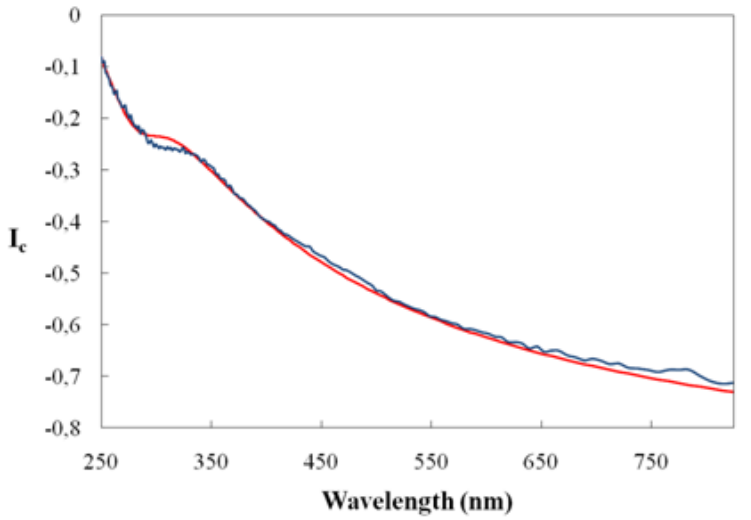

\begin{tabular}{|l|l|}
\hline Ir & void \\
\hline
\end{tabular}

Ir

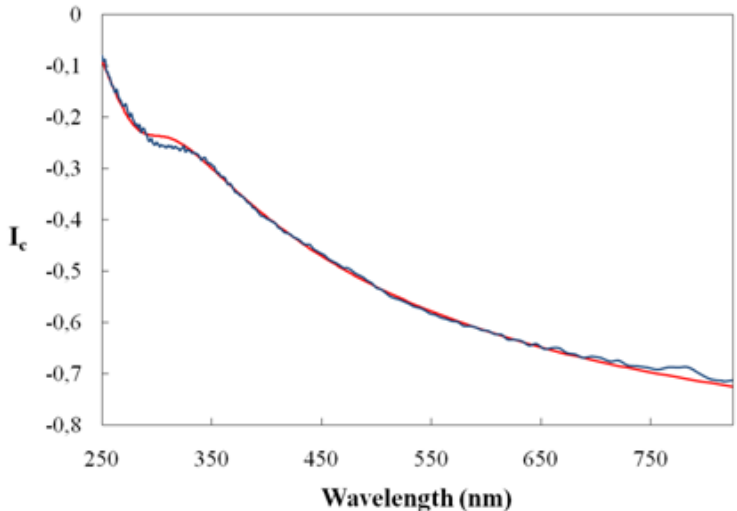

$\mathrm{a}-\mathrm{C}$ Ir

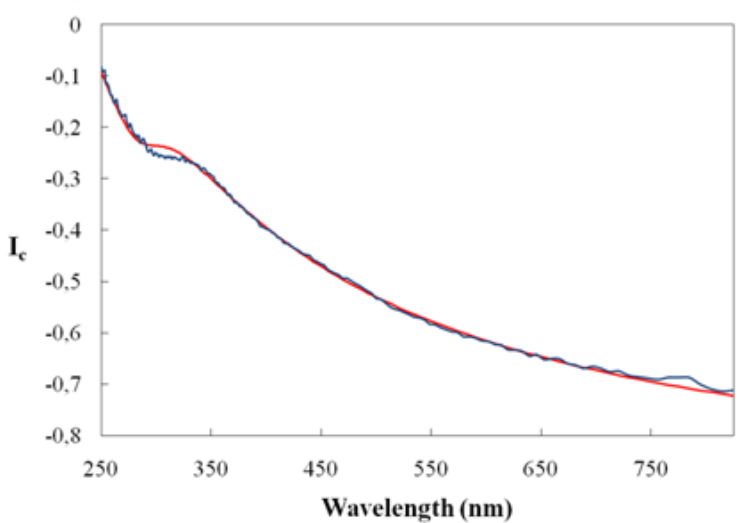

Fig. 5. Comparison of $I_{s}$ and $I_{c}$ intensities extracted from models (in red) and experimental data (in blue) in a 250$825 \mathrm{~nm}$ wavelength range. The three models represent (a) the formation of domains without the iridium roughening, (b) the roughening of iridium surface without the formation of domains and (c) a combination of both phenomena. 
Fig. 5c shows the comparison of $I_{s}$ and $I_{c}$ intensities extracted from the third model after optimization with the experimental acquisitions. The obtained $\chi^{2}$ is similar to the previous model $(0.48)$ and it is not sufficient to select the model relevance. Using this third model, the first layer is composed of $65 \pm 22$ $\%$ and $35 \pm 22 \%$ of amorphous carbon and iridium, respectively, it is $1.4 \pm 0.2 \mathrm{~nm}$ thick. Amorphous carbon composes $57 \pm 19 \%$ of the second layer which is $1.4 \pm 0.4 \mathrm{~nm}$ thick. This leads to a mean thickness of $1.7 \pm 0.4 \mathrm{~nm}$ for the amorphous carbon.

\subsection{Annealing of the sample after the BEN step}

During the BEN step, two phenomena are competing: the roughening of the iridium surface and the formation of domains. It is essential to ensure that the SE enables the detection of one phenomenon or the other distinctly. A surface treatment that removes domains without changing the iridium morphology was applied and SE characterization was performed before and after. For this work, the 10x10 mm² $\mathrm{Ir} / \mathrm{SrTiO}_{3} / \mathrm{Si}(001)$ pseudo-substrate underwent the BEN step. After BEN, an annealing under air at $450^{\circ} \mathrm{C}$ during $1 \mathrm{~h}$ was applied to the sample to remove the carbon layer. Fig. 6 shows FESEM images exactly of the same area of the sample after the BEN step and after the annealing. On Fig. 6a, domains are observable as well as iridium balls and furrows (encircled). After the air annealing, FE-SEM images don't present areas with a bright contrast: domains were totally removed (Fig. 6b). However, iridium balls and furrows are still present on the surface. This shows the negligible effect of the annealing on the iridium surface morphology. 
(a)

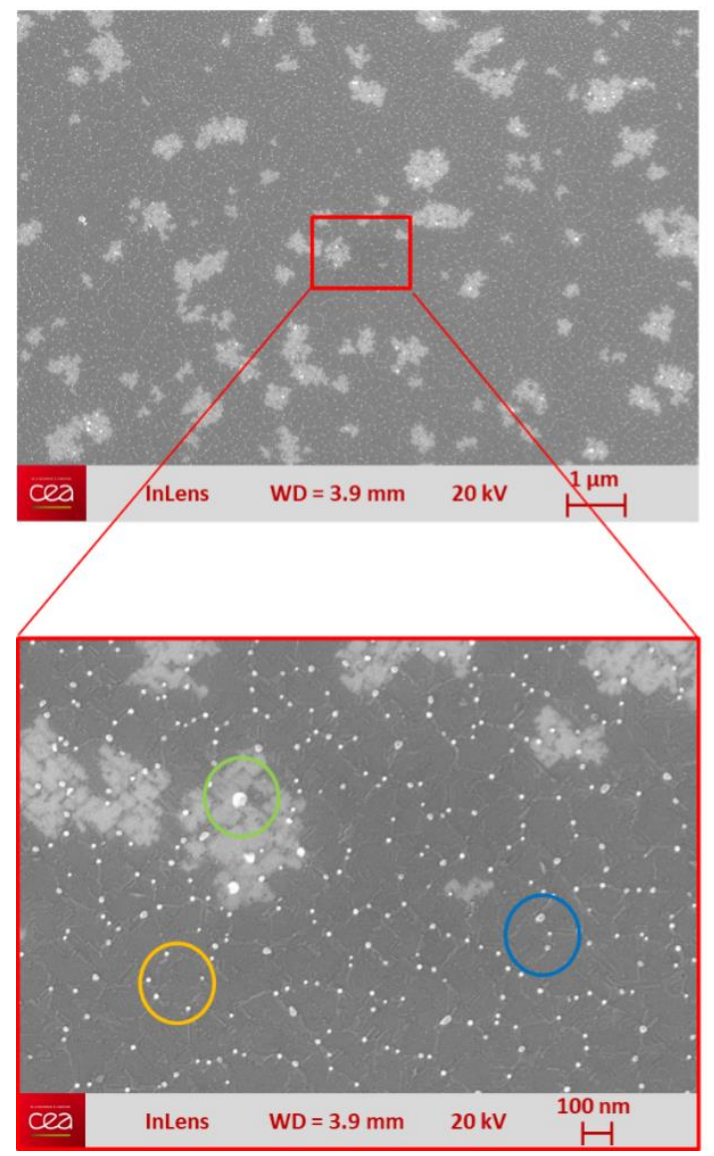

(b)

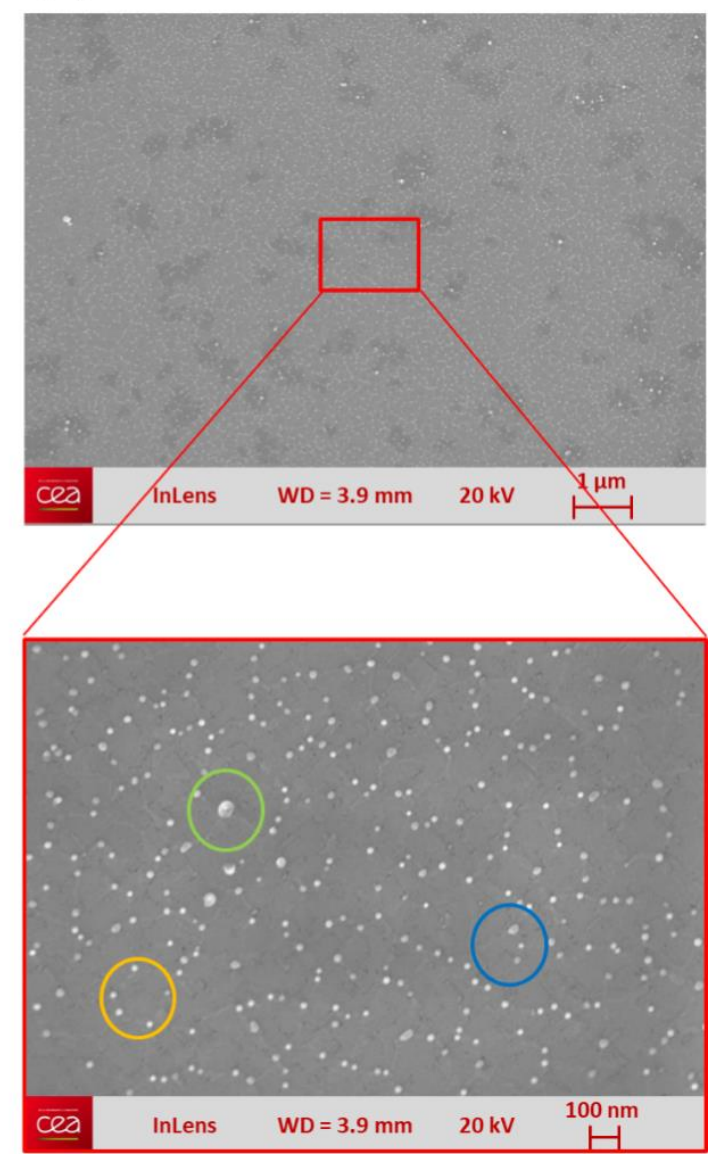

Fig. 6. FE-SEM images recorded exactly on the same area of the iridium surface (a) after the nucleation step and (b) after the air annealing treatment.

$\mathrm{I}_{\mathrm{s}}$ and $\mathrm{I}_{\mathrm{c}}$ intensities were acquired by SE after the iridium deposition, after the BEN step and after the air annealing on the same area of the sample. The reproducibility of the SE measurement was measured doing several measurements on the same area. Before each measurement, the sample was removed and replaced in the same geometrical configuration. Results obtained for SE acquisitions after the BEN step and after the annealing are illustrated in Fig. 7 (blue and green curves respectively). After each measurement, the spectra superimposed very well; the limitation is the noise of the signal that is around 0.003 .

The comparison of signals on a 250-825 nm wavelength range is presented in Fig. 7. After the BEN step, $I_{s}$ and $I_{c}$ intensities increase, especially from $350 \mathrm{~nm}$ for $I_{s}$. After the air annealing, $I_{s}$ decreases by around 0.015 from $300 \mathrm{~nm}$ for $\mathrm{I}_{\mathrm{s}}$ and around 0.030 from $250 \mathrm{~nm}$ for $\mathrm{I}_{\mathrm{c}}$; The artifact at $520 \mathrm{~nm}$ is 
much higher for the initial iridium surface because the ellipsometric spectrometer was recalibrated before the BEN step.
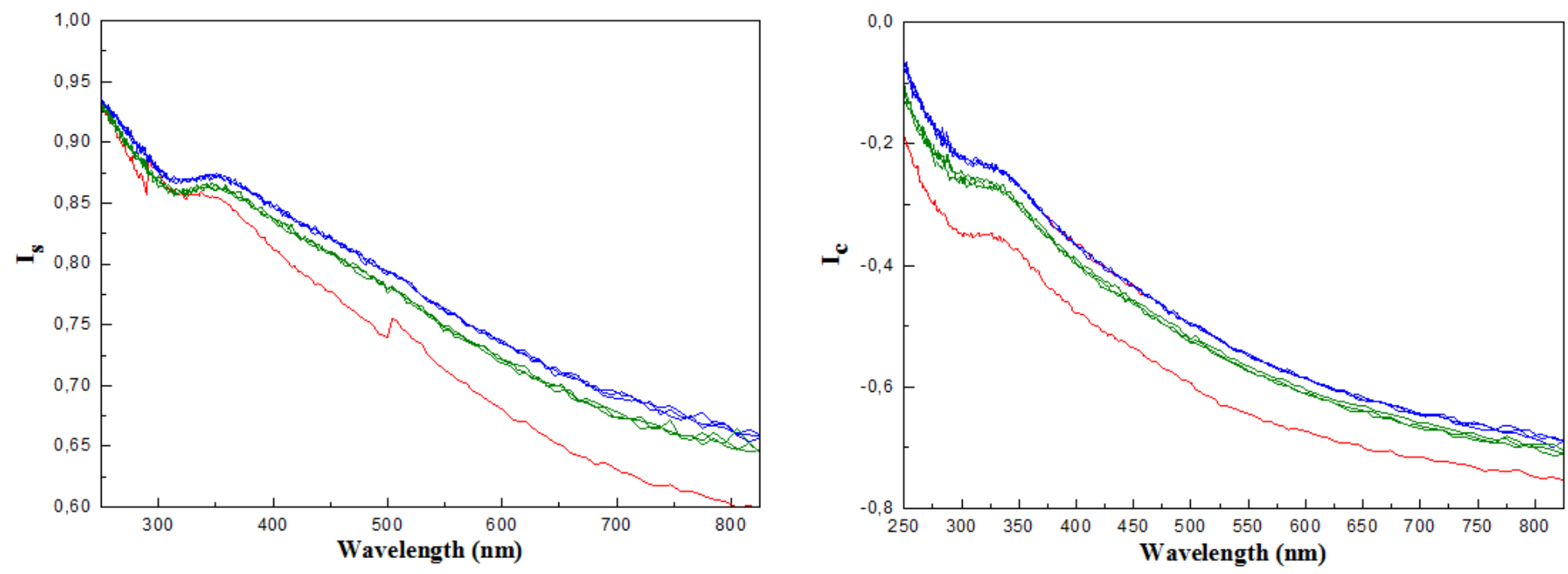

Fig. 7. Comparison of $I_{s}$ and $I_{c}$ intensities experimentally acquired by $S E$ on the initial iridium surface (in red), several measurements after the BEN step (in blue) and after the annealing treatment (in green).

\section{Discussion}

\subsection{Sequential analysis and conception of an ellipsometric model}

The two first proposed models present a low value of $\chi^{2}(<1)$. Nevertheless, the visible difference between the experimental data and simulations for $I_{s}$ and $I_{c}$ shows the necessity to take into account both phenomena, i.e. roughening and domain formation to build a more robust model conserving a physical meaning. In this third model, diamond nuclei included in domains were not considered. Indeed, N. Vaissiere et al. demonstrated with combined characterizations by FE-SEM, XPS and NanoAuger spectroscopy of domains that diamond nuclei are embedded in the amorphous carbon layer [15]. The final model allows the measurement of a coverage ratio of domains (57\%) well correlated with the value deducted from the statistical analysis of thirty FE-SEM images (44\%). We also deduced a thickness of the rough iridium surface of $1.7 \mathrm{~nm}$ that is well correlated with the RMS roughness measured by AFM $(2.4 \mathrm{~nm})$. Moreover, this model leads to a mean thickness for the carbon layer in the same order of magnitude compared to the value calculated with XPS analysis $(1.7 \mathrm{~nm}$ and $4.6 \mathrm{~nm}$, respectively). Note that the amorphous carbon layer estimated from XPS analysis was obtained 
assuming a homogeneous carbon layer that does not represent the reality. Nevertheless, we can deduce that the thickness of domains is included between $1.4 \pm 0.2 \mathrm{~nm}$ and $2.8 \pm 0.6 \mathrm{~nm}$ taking into account the two amorphous carbon layers of the model which is in accordance with the literature. Also, this third model does not contradict the fact that the whole iridium surface is covered by a thin layer of amorphous carbon as shown by M. Schreck et al. [11]. Nevertheless, this model is more complex because of its multi-layer architecture and consequently, the number of parameters used for fitting is high. Thus, it is not enough reliable to separate the contribution of iridium surface roughening from amorphous carbon deposition and to be used as an in situ tool to probe the domains formation. To check if the SE allows the decorrelation of both phenomena, we performed a thermal treatment to remove domains without changing the iridium surface morphology. SE data recorded before and after the annealing were then compared on the same area.

\subsection{Annealing after the BEN step and sequential analysis by ellipsometry}

SE acquisitions made on the initial iridium surface, after the BEN step and after the annealing demonstrate the possibility to detect the roughening of the iridium surface (iridium balls and furrows), and the formation of domains distinctly. Indeed, the gap between the red and green curves in Fig. 7 represents the contribution of the formation of iridium balls and furrows in the ellipsometric intensities $I_{s}$ and $I_{c}$. The gap between the green and blue curves represents the contribution of domains. Even if the difference between these two curves for $I_{s}$ and $I_{c}$ intensities are only five and ten times higher than the noise of the signal, respectively, this study constitutes the first step towards in situ analysis of the domains formation. Same measurements should be performed at the temperature under which the diamond nucleation occurs, i.e. $700-800^{\circ} \mathrm{C}$. Indeed, the optical properties $(\mathrm{n}, \mathrm{k})$ are temperature dependent. If this stage is validated, SE could be used in situ to monitor the early stages of BEN which would be pioneering. This technique may accelerate the finding of BEN parameters on 2 in. $\mathrm{Ir} / \mathrm{SrTiO}_{3} / \mathrm{Si}(001)$ wafers and deepen our knowledge of mechanisms induced by the BEN step resulting in the formation of domains.

\section{Conclusion}

The complex evolution of the iridium surface occurring during the BEN with mechanisms competing with heteroepitaxial diamond nucleation explains the high interest for in situ monitoring. This approach could lead to an accurate optimization of the BEN parameters for diamond heteroepitaxy on 
large $\mathrm{Ir} / \mathrm{SrTiO}_{3} / \mathrm{Si}(001)$ wafers (2 in. or more). Such experimental approach will also deepen the knowledge in the formation of domains. Considering its sensitivity to the optical index differences at interfaces, SE was investigated. Sequential analysis by FE-SEM, AFM and XPS was performed on substrate with domains in order to build the most relevant ellipsometric model keeping a physical meaning. The comparison between the ellipsometric analysis and the model showed the possibility to characterize the iridium surface on which coexist iridium balls, furrows and domains. However, the proposed model is not reliable enough to decorrelate the contribution from iridium roughening to the one related to domain formation. To verify the possibility to detect the roughening of the iridium surface and the formation of domains distinctly, we performed an air annealing treatment at $450^{\circ} \mathrm{C}$ to remove domains. With FE-SEM characterizations exactly performed on the same area of the surface, we demonstrated that domains are totally removed without changing the iridium surface morphology. Finally, sequential analysis by SE proved its sensitivity to the formation of domains. This result opens the way to in situ monitoring of early stages of heteroepitaxial diamond growth during BEN, which would allow enhancing the crystalline quality of heteroepitaxial diamond layers for various key applications.

\section{Acknowledgments}

Authors would like to thank the French National Agency (ANR) and DGA for funding this work under the project DIAMWAFEL (Large and conducting DIAMondWaFers for industrial applications in power ELectronics), Grant No. ANR-15-CE08-0034. Authors would like to acknowledge Nicolas Tranchant for useful discussions.

\section{References}

[1] N. Donato, N. Rouger, J. Pernot, G. Longobardi, F. Udrea, Diamond power devices: state of the art, modelling, figures of merit and future perspective, J. Phys. D: Appl. Phys. 53 (2020) 093001

[2] M. Schreck, J. Asmussen, S. Shikata, J.C. Arnault, N. Fujimori, Large-area high quality single crystal diamond, MRS Bulletin. 39 (2014) 504-510. doi:10.1557/mrs.2014.96. 
[3] K. Ichikawa, K. Kurone, H. Kodama, K. Suzuki, A. Sawabe, High crystalline quality heteroepitaxial diamond using grid patterned nucleation and growth on Ir, Diamond and Related Materials. 94 (2019) 92.

[4] J.C. Arnault, K.H. Lee, J. Delchevalrie, J. Penuelas, L. Mehmel, O. Brinza et al., Epitaxial diamond on Ir/ SrTiO3/Si (001): From sequential material characterizations to fabrication of lateral Schottky diodes, Diamond and Related Materials. 105 (2020) 107768.

[5] M. Schreck and J. C. Arnault, Heteroepitaxy of diamond on Ir/metal-oxide/ Si substrates in Power Electronics Device Applications of Diamond Semiconductors, S. Koizumi, H. Umezawa, J. Pernot and M. Suzuki Eds, Elsevier (2019) ISBN: 9780081021835

[6] K. H. Lee, S. Saada, J. C. Arnault, R. Moalla, G. Saint-Girons, R. Bachelet et al., Epitaxy of iridium on $\mathrm{SrTiO}_{3} / \mathrm{Si}$ (001): A promising scalable substrate for diamond heteroepitaxy, Diam. Rel. Mater. 66 (2016) 67.

[7] H. Liu, D. S. Dandy, Studies on nucleation process in diamond CVD: an overview of recent developments, Diamond and Related Materials. 4 (1995) 1173-1188.

[8] J.C. Arnault, S. Saada, S. Delclos, L. Intiso, N. Tranchant, R. Polini et al., In situ study of the initial stages of diamond deposition on 3C-SiC (100) surfaces: Towards the mechanisms of diamond nucleation, Diam. Relat. Mater. 16 (2007) 690-694.

[9] F. Hörmann, M. Schreck, B. Stritzker, First stages of diamond nucleation on iridium buffer layers, Diamond and Related Materials. 10 (2001) 1617-1621.

[10] M. Schreck, Th. Bauer, S. Gsell, F. Hörmann, H. Bielefeldt, B. Stritzker, Domain formation in diamond nucleation on iridium, Diamond and Related Materials. 12 (2003) 262-267.

[11] M. Schreck, S. Gsell, R. Brescia, M. Fisher, Ion bombardment induced buried lateral growth: the key mechanism for the synthesis of single crystal diamond wafers. Sci Rep 7, 44462 (2017).

[12] J. C. Arnault, L. Intiso, S. Saada, S. Delclos, P. Bergonzo, R. Polini, Effect of 3C-SiC (100) initial surface stoichiometry on bias enhanced diamond nucleation, Appl. Phys. Lett. 90 (2007) 044101. 
[13] A. Chavanne, J.-C. Arnault, J. Barjon, J. Arabski, Bias-enhanced nucleation of diamond on iridium: A comprehensive study of the first stages by sequential surface analysis, Surface Science. 605 (2011) 564-569.

[14] S. Saada, S. Pochet, L. Rocha, J.C. Arnault, P. Bergonzo, Real time investigation of diamond nucleation by laser scattering, Diamond and Related Materials. 18 (2009) 707-712.

[15] N. Vaissiere, S. Saada, M. Bouttemy, A. Etcheberry, P. Bergonzo, J.C. Arnault, Heteroepitaxial diamond on iridium: new insights on domain formation, Diam. Relat. Mater. 36 (2013) 16.

[16] F. Hörmann, Th. Bauer, M. Schreck, S. Gsell, B. Stritzker, TEM analysis of nanometer-size surface structures formed by bias enhanced nucleation of diamond on iridium, Diamond and Related Materials. 12 (2003) 350-355.

[17] J. Bousquet, G. Chicot, D. Eon, E. Bustarret, Spectroscopic ellipsometry of homoepitaxial diamond multilayers and delta-doped structures, Appl. Phys. Lett. 104 (2014) 021905

[18] G. Niu, G. Saint-Girons, B. Vilquin, G. Delhaye, J.-L. Maurice, C. Botella et al., Molecular beam epitaxy of SrTiO3 on Si (001): Early stages of the growth and strain relaxation, Applied Physics Letters. 95 (2009) 062902.

[19] G. Niu, B. Vilquin, J. Penuelas, C. Botella, G. Hollinger, G. Saint-Girons, Heteroepitaxy of SrTiO3 thin films on Si (001) using different growth strategies: Toward substrate like quality, Journal of Vacuum Science \& Technology B: Microelectronics and Nanometer Structures. 29 (2011) 041207.

[20] L. Louahadj, Développement de l'épitaxie par jet moléculaire d'oxydes fonctionnels sur silicium, $\mathrm{PhD}$, Ecole Centrale de Lyon, 2014.

[21] Y. Wei, X. Hu, Y. Liang, D.C. Jordan, B. Craigo, R. Droopad, et al., Mechanism of cleaning $\mathrm{Si}(100)$ surface using $\mathrm{Sr}$ or $\mathrm{SrO}$ for the growth of crystalline $\mathrm{SrTiO}_{3}$ films, Journal of Vacuum Science \& Technology B. 20 (2002) 1402. 
[22] J. Delchevalrie, Films de diamant hétéroépitaxiés sur $\mathrm{Ir} / \mathrm{SrTiO} 3 / \mathrm{Si}(001)$ : une voie vers des substrats de plus grande taille, PhD, Université Paris Saclay, 2019

[23] A. Chavanne, J. Barjon, B. Vilquin, J. Arabski, J.C. Arnault, Surface investigations on different nucleation pathways for diamond heteroepitaxial growth on iridium, Diamond and Related Materials. 22 (2012) 52-58.

[24] D. E. Aspnes, Spectroscopic ellipsometry - Past, present, and future, Thin Solid Films. 571 (2014) 334-344.

[25] A. Yanguas-Gil, H. Wormeester, Relationship between surface morphology and effective medium roughness, in: M. Losurdo, K. Hingerl (Eds.), Ellipsometry at the Nanoscale, Springer, Berlin Heidelberg, Berlin, Heidelberg 2013, pp. 179-202. 